\title{
The Economics of NASA Mission Cost Reserves
}

\author{
Sally Whitley \\ The Johns Hopkins University Applied Physics \\ Laboratory \\ 11100 Johns Hopkins Rd. \\ LaureI, MD 20723 \\ 443-778-4179 \\ sally.whitley@jhuapl.edu
}

\begin{abstract}
Increases in NASA mission costs are well-noted but not well-understood, and there is little evidence that they are decreasing in frequency or amount over time (Bitten and Freaner, 2010). The need to control spending has led to analysis of the causes and magnitude of historical mission overruns, and many program control efforts are being implemented to attempt to prevent or mitigate the problem (NPR 7120). However, cost overruns have not abated, and while some direct causes of increased spending may be obvious (requirements creep, launch delays, directed changes, etc.), the underlying impetus to spend past the original budget may be more subtle. Gaining better insight into the causes of cost overruns will help NASA and its contracting organizations to avoid them.
\end{abstract}

This paper hypothesizes that one cause of NASA mission cost overruns is that the availability of reserves gives project team members an incentive to make decisions and behave in ways that increase costs. We theorize that the presence of reserves is a contributing factor to cost overruns because it causes organizations to use their funds less efficiently or to control spending less effectively. We draw a comparison to the insurance industry concept of moral hazard, the phenomenon that the presence of insurance causes insureds to have more frequent and higher insurance losses, and we attempt to apply actuarial techniques to quantify the increase in the expected cost of a mission due to the availability of reserves.

We create a theoretical model of reserve spending motivation by defining a variable ReserveSpending as a function of total reserves. This function has a positive slope; for every dollar of reserves available, there is a positive probability of spending it. Finally, the function should be concave down; the probability of spending each incremental dollar of reserves decreases progressively. We test the model against available NASA CADRe data by examining missions with reserve dollars initially available and testing whether they are more likely to spend those dollars, and whether larger levels of reserves lead to higher cost overruns.

Finally, we address the question of how to prevent reserves from increasing mission spending without increasing cost risk to projects budgeted without any reserves. Is there a "sweet spot"? How can we derive the maximum benefit associated with risk reduction from reserves while minimizing the effects of reserve spending motivation?

\author{
Stephen Shinn \\ NASA Goddard Space Flight Center \\ 8800 Greenbelt Road \\ Greenbelt, MD 20771 \\ 301-286-5894 \\ stephen.a.shinn@nasa.gov
}

TABLE OF CONTENTS

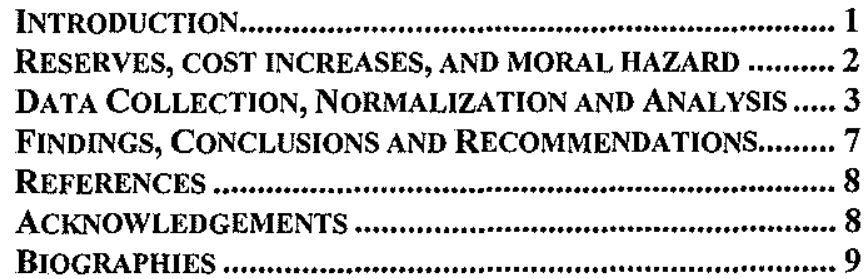

\section{INTRODUCTION}

Increases in NASA mission costs are well-noted but not well-understood, and there is little evidence that they are decreasing in frequency or amount over time (Bitten and Freaner, 2010). The need to control spending has led to analysis of the causes and magnitude of historical mission overruns, and many program control efforts are being implemented to attempt to prevent or mitigate the problem (NPR 7120). Results of these measures are only now becoming available for study, since the travel time from establishment to enforcement to outcome of such requirements is years. Evidence from the most recent mission cost data shows that the new reporting and control requirements have produced indifferent results at best. For example, Shinn et al. (2011) found that the magnitude of mission cost increases, measured as change in cost between successive milestones included in CADRe data, has no apparent correlation with launch year. At worst, the new requirements may be so cumbersome that they cost more to implement and enforce than they save. Some preliminary findings indicate that one cause of mission cost increases could be that costs associated with program management and systems engineering are estimated from historical data to which less onerous requirements applied. This bias causes current programs to underestimate effort associated with these activities unless historical data are adjusted accordingly (Shinn et al. 2011).

As the example above demonstrates, better insight into the causes of cost overruns helps NASA and its contracting organizations to avoid them by improving cost estimating methods. However, cost overruns occur across all elements of the NASA WBS, and the data show that overruns are more common in spacecraft and payload than in PM and SE (Figure 1). While some direct causes of increased spending may be obvious (requirements creep, launch delays, directed changes, etc.), some examples of the impetus to spend past the original budget may be more subtle. For example, 
suppose a mission experienced cost growth of $36 \%$ between its PDR and its launch. If it fell under the requirements of NPR $7120.5 \mathrm{C}$, had no launch delay, and reports directed changes of only $10 \%$, where did the remaining $26 \%$ increase come from?

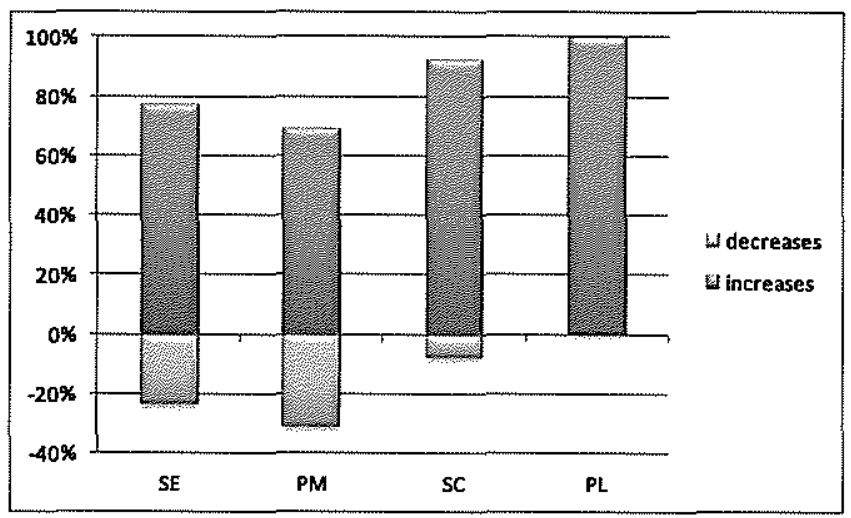

Figure 1. Frequency of Cost Increases by WBS for NASA Missions in CADRe Data Set

Several studies have been commissioned by NASA to evaluate the explanation of cost and schedule changes throughout the mission life cycle. Requirements growth, technical complexity, re-work, and inherent optimism are often cited as primary causes of cost increases. Funding instability can also play a factor in work authorization, causing projects to constantly re-plan efforts. In many of these cases, the "marching army" cost can increase as projects are pushed forward in time. Could intangible factors, such as political environment or knowledge of stakeholder intentions also play a role? It has been widely discussed that contractors behave differently when they understand how much money in total their customer may have for a project. Could the same phenomenon occur within the NASA community?

This paper hypothesizes that one cause of NASA mission cost overruns is that the availability of reserves gives project team members an incentive to make decisions and behave in ways that increase costs. We theorize that the presence of reserves is a contributing factor to cost overruns because it causes organizations to use their funds less efficiently or to control spending less effectively. We draw a comparison to the economic concept of moral hazard, the phenomenon that the presence of insurance (or any form of contractually guaranteed loss-indemnification) causes insureds to have more frequent and higher insurance losses, (Mirrlees, 1999).

\section{RESERVES, COST INCREASES, AND MORAL HAZARD}

The terms reserve, management reserve, and project reserves are often used interchangeably in the aerospace environment. However, they have very different meanings and structure. The planning, use, and application of each term varies significantly. We will briefly discuss these terms and approach, working from the lowest level application to the highest level discussion. A basic tenet of sound project management is that a project manager should hold reserve to accommodate "known-unknowns." That is, every project will encounter anticipated costs that cannot yet be specifically identified during the planning process. In the Earned Value Management (EVM) context, this type of contingency is known as Management Reserve (MR). Management reserve is defined as "An amount of total allocated budget withheld for management control purposes rather than designated for the accomplishment of a specific task or set of tasks." (cited). The fundamental difference with this type of reserve is that it is held by the project and carved from the existing project contract budget base. It is wholly controlled by the project manager and can be allocated to specific control accounts via a baseline change request. The reserves and project-reserves discussed in this paper are the higher level holdings that are allocated in addition to what the project has requested (project budget). That is, the reserves are over and beyond the grass roots or parametric cost estimates generated for the completion of a specific mission. The NASA 7120.5D document describes project reserves management as "one of the most important functions of project management." In this context, reserves are typically applied as a \% or allocation over and above the project baseline estimate. However, these reserve amounts are readily known, spent, and understood by project teams. Further, the NASA 7120 handbook actually provides insights and recommendations for properly planning and expending reserves dollars. Our assertion is that this can actually exacerbate cost overruns based on the project's knowledge of a "safety net."

Moral hazard arises when an entity changes its behavior because it does not bear the full consequences of the risk that it takes on. An example is the statistical correlation between automobile insurance and auto theft. Insured cars are more likely to be stolen than uninsured cars. One reason for this is that automobile owners who have insurance are more likely to behave carelessly about locking their cars due to the presence of the insurance. This phenomenon can be found in many different types of risk transfer arrangements, including banking transactions (e.g., the savings and loan losses of the mid-eighties), stock brokering, demand for medical services by insured patients, and long-term disability "malingering." Many risk transfer contracts include risk sharing provisions that force the riskadvantaged entity to partake in the losses of the riskdisadvantaged entity to an extent commensurate with the expected losses associated with moral hazard. Insurance deductibles, medical co-pays and performance-based commission are all examples of risk-sharing arrangements to counter moral hazard.

In the aerospace industry, cost reserves are intended, ideally, to absorb risks associated with estimating space mission costs years before launch. Estimate uncertainty is an accepted part of the planning process, and reserves provide a form of insurance against cost overruns. They protect both 
NASA and its contracting organizations from mission failure caused by unexpected expenses. However, they may also create incentives akin to moral hazard because both the contracting organization and NASA have less reason to worry about unsustainable cost growth. Since we noted above that cost growth can occur for reasons within, as well as for reasons outside of a project's control, reserves may lead to increases in spending that are not necessarily associated with unforeseen project risks, but rather with cost deltas that could otherwise be mitigated or avoided.

Consider the following anecdotal example of a husband and wife planning a kitchen remodeling project. In one scenario, they agree that their budget for the renovation is $\$ 20,000$, but they will set aside $\$ 5,000$ ( $25 \%$ reserves) to cover unknowns. In a second scenario, their budget is also $\$ 20,000$, but they don't have any additional funds for overages. In fact, if they go over the $\$ 20,000$ limit, there is a high likelihood that they will be unable to cover their mortgage payments and risk losing the house. It is clear that the former example has a much greater chance of overrun than the latter. We contend that the knowledge of reserves could certainly play a role in the spending of reserves.

In order to establish whether mission cost reserves constitute a self-fulfilling prophecy, we need to construct a theory and test it against available data. A theoretical model of reserve spending motivation defines a variable ReserveSpending as a function of total reserves.

\section{Equation 1}

$$
\text { ReserveSpending }=f(\text { Reserves })
$$

This function has a positive slope; for every dollar of reserves available, there is a positive probability of spending it.

$$
\frac{\text { SReserveSpending }}{\delta \text { Reserves }}>0 \text { for all Reserves }>0
$$

Equation 2

Finally, the function should be concave down; the probability of spending each incremental dollar of reserves decreases progressively (Equation 3 ).

$$
\frac{\delta^{2} \text { ReserveSpending }}{\delta \text { Reserves }^{2}}<0 \text { for all Reserves }>0
$$

\section{Equation 3}

The first model requirement (Equation 1), states that the amount of reserves that a mission spends is a function of the amount of reserves that are available to the mission. The model does not require that reserves are the only factor contributing to reserve spending, as we have already shown above. However, we do hypothesize that the presence or absence of reserves affects the probability of spending beyond initial mission cost estimates. The second model requirement (Equation 2) states that the relationship between reserves and reserve spending is positive. Here we hypothesize that larger reserves lead to larger cost increases. The more reserve dollars are made available to cover mission costs, the greater the cost growth associated with the mission. Finally, Equation 3 hypothesizes that cost growth is throttled as reserve dollars are successively spent. As reserves begin to run out, we expect to see slow-down in cost growth. In other words, the first dollar of reserves is the most likely one to be spent on cost increases, and the last dollar of reserves is the least likely.

We first tested the model against available NASA CADRe data. We tried to examine missions with reserve dollars initially available and missions to which no reserves applied. We tested whether reserved missions are more likely to experience mission cost growth between successive milestones, and whether larger levels of reserves lead to higher cost overruns. Finally, we tested whether reserve spending slows as reserve dollars are depleted.

\section{Data Collection, Normalization AND ANALYSIS}

We examined all available CADRe data to collect a data set on which to test our hypothesis. The challenge was, as always, getting consistent, reliable data. Several requirements for our data set were:

1. Missions included require both a PDR CADRe and a launch CADRe.

2. Cost data are required for total mission, spacecraft, payload, program management and systems engineering.

3. Launch vehicle costs must be broken out.

4. Reserves must be broken out.

Anomalous data were excluded from our analysis. The final data set included 13 data points for the following missions:

Table 1. CADRe Missions Included in this Analysis

\begin{tabular}{|l|l|l|}
\hline AIM & CLOUDSAT & DAWN \\
\hline GLAST & IBEX & LRO \\
\hline MESSENGER & MRO & New Horizons \\
\hline PHOENIX & STEREO & CALIPSO \\
\hline Spitzer & & \\
\hline
\end{tabular}

We defined total cost overrun (which can be negative) as the change in mission cost between CDR and launch, excluding reserves and launch vehicle. Since we also collected various WBS elements in isolation, we defined cost increases for program management, systems engineering, spacecraft, and payload similarly, as the change in reported costs for each of these WBS elements between PDR and launch. Reserves were defined as given by the CADRe in a separate line item (sometimes referred to as "contingency").

Upon finalization of the data set, it became clear that the first part of our hypothesis, that reserved missions are more likely to experience cost overruns than unreserved missions, 
would not be testable using available CADRe data. The reason for this is that all of the missions in our data set had reserves at PDR. We were unable to find an example of a mission with CADRes at both PDR and launch for which the cost estimate did not set aside reserves at PDR.

The next part of our hypothesis, however, was testable. We examined whether mission cost increases correlate positively with reserve levels. A first look at total mission cost increase as a percentage of PDR cost estimate (excluding reserves and launch vehicle) against reserve percentage revealed no statistical significance (Figure 2). We encountered similarly inconclusive findings for spacecraft (Figure 3), payload (Figure 4), program management (Figure 5), and systems engineering (Figure 6) increases when taken as a percentage of the initial estimate.

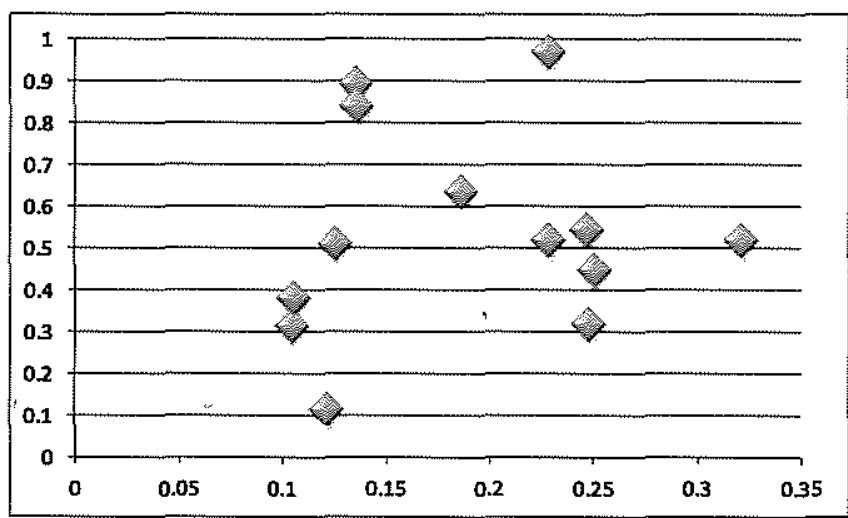

Figure 2. Total Mission Cost Increase Against Reserves, Both as Percentages of Mission Costs Estimated at PDR, Exclusive of Launch Vehicle and Reserve

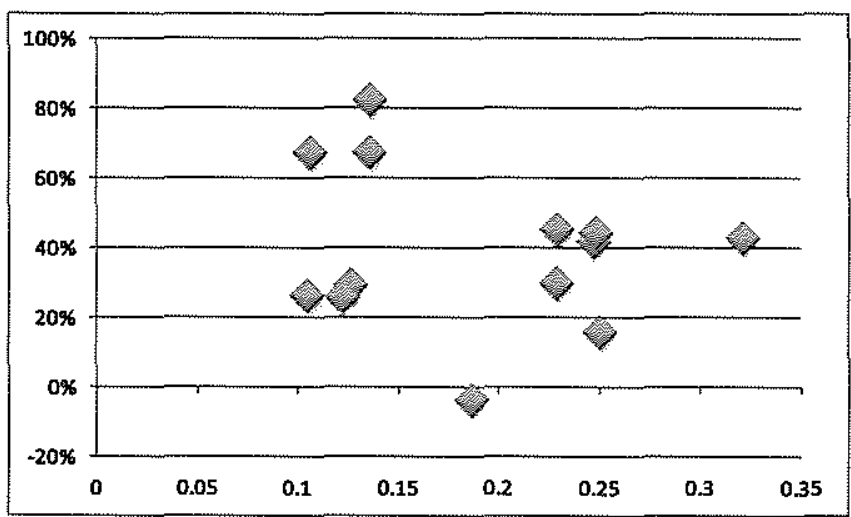

Figure 3. Percentage of Increase in Spacecraft Costs Against Percentage Reserves

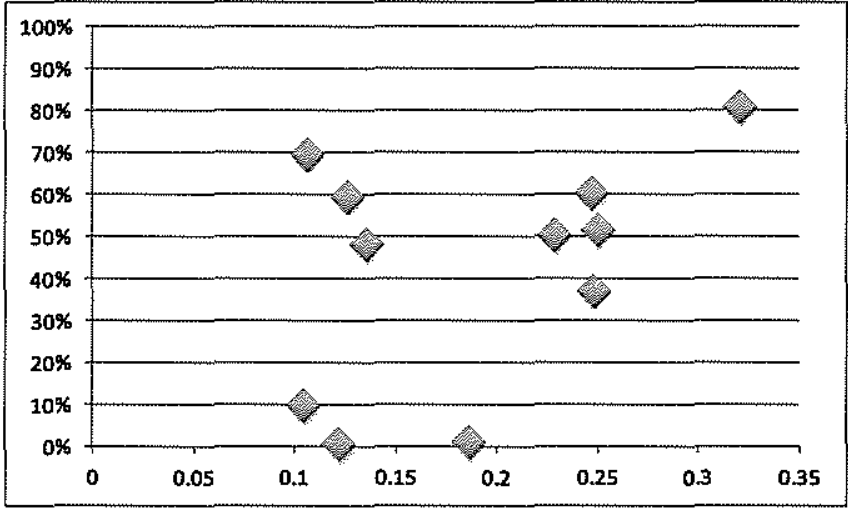

Figure 4. Percentage of Increase in Payload Costs Against Percentage Reserve

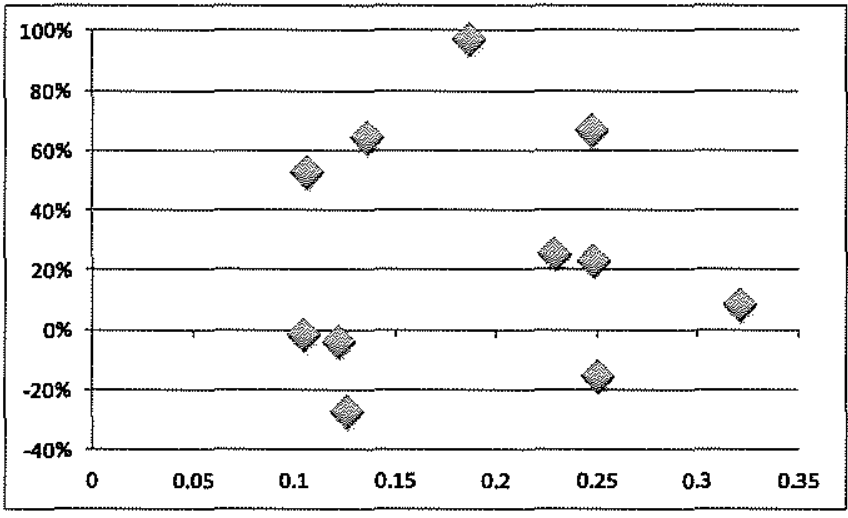

Figure 5. Percentage Program Management Cost Increase Against Percentage Reserve

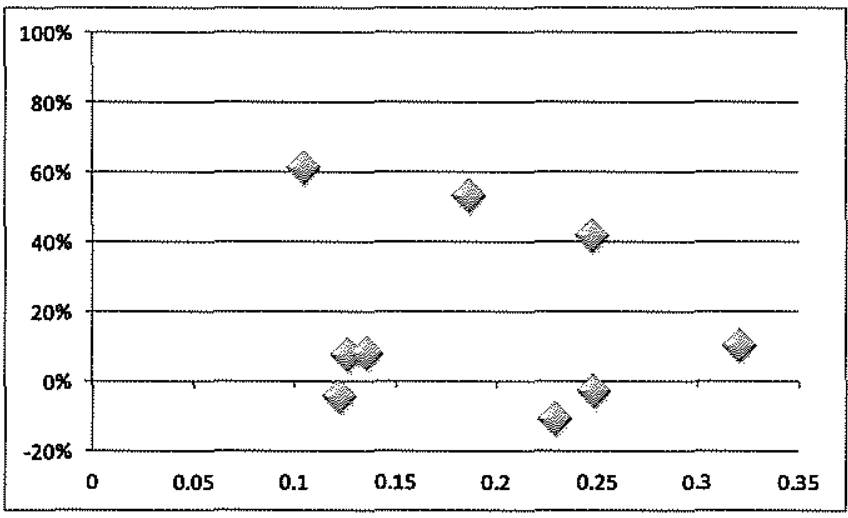

Figure 6. Percentage Systems Engineering Cost Increase Against Percentage Reserve

We next examined whether total dollars spent bore a positive relationship with total dollars reserved for each of the cost groups. Findings demonstrated strong positive correlation between total mission cost increases and total dollars reserved, as shown in Figure 7. The same was true for hardware cost increases, as shown for spacecraft in Figure 8, and for payload in Figure 9. Figure 10 shows the 
relationship between total cost increase of spacecraft plus payload as a function of total dollars of reserves.

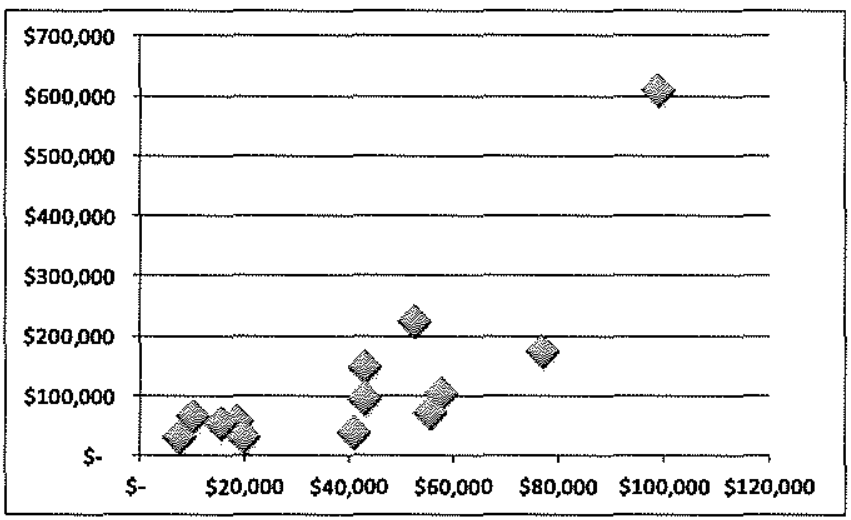

Figure 7. Total Dollars of Mission Cost Increase Against Total Dollars Reserve

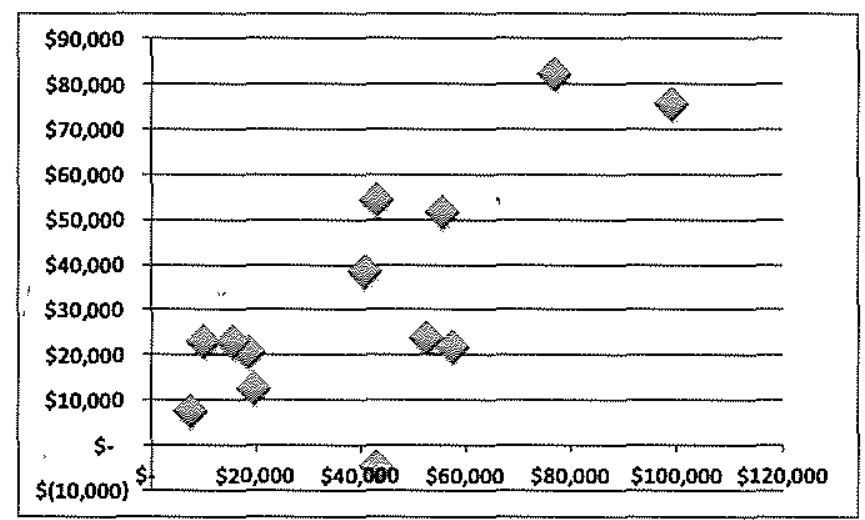

Figure 8. Total Dollars of Spacecraft Cost Increase Against Total Dollars Reserved

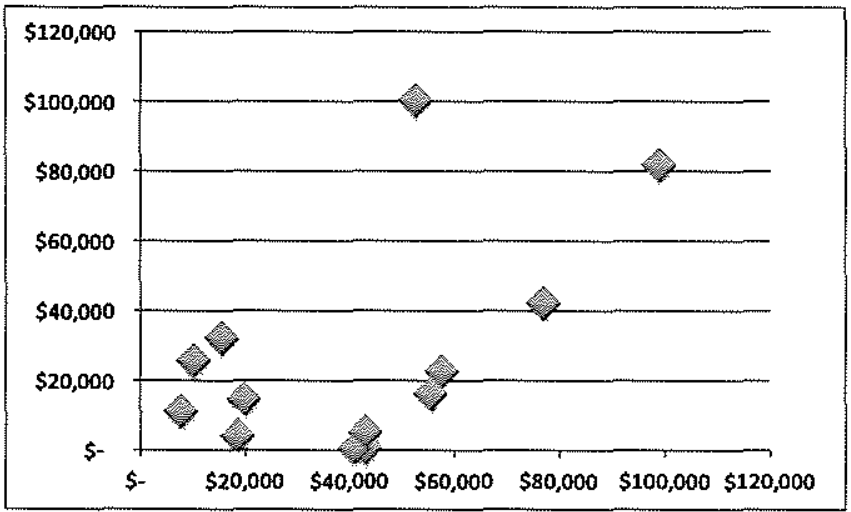

Figure 9. Total Dollars of Payload Cost Increase Against Total Dollars of Reserves

No such strong relationship could be found between reserved dollars and cost increases in program management (Figure 11) and systems engineering (Figure 12).

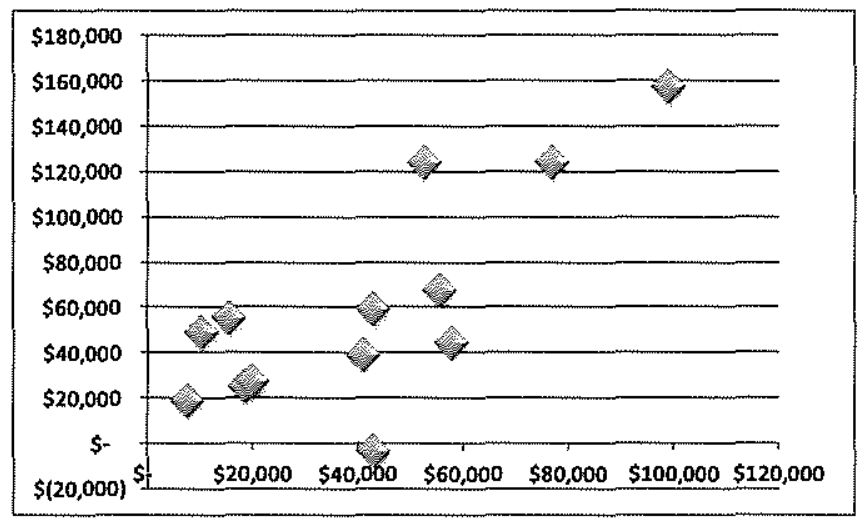

Figure 10. Total Dollars of Spacecraft Plus Payload Cost Increase Against Total Dollars of Reserves

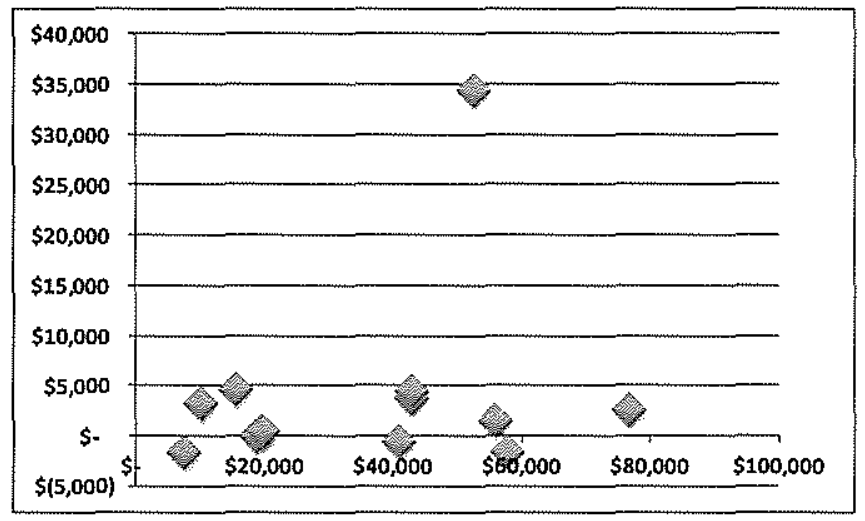

Figure 11. Total Dollars of Program Management Cost Increase Against Total Dollars of Reserves

Having established a strong positive relationship between total dollars of mission cost increase and total dollars of reserves, we tested the third part of our hypothesis. The question was whether there is evidence that cost increases tend to slow down as reserve dollars are used up. We examined cost increases as a percentage of mission reserves for each of the categories, total mission, spacecraft, payload, program management and systems engineering.

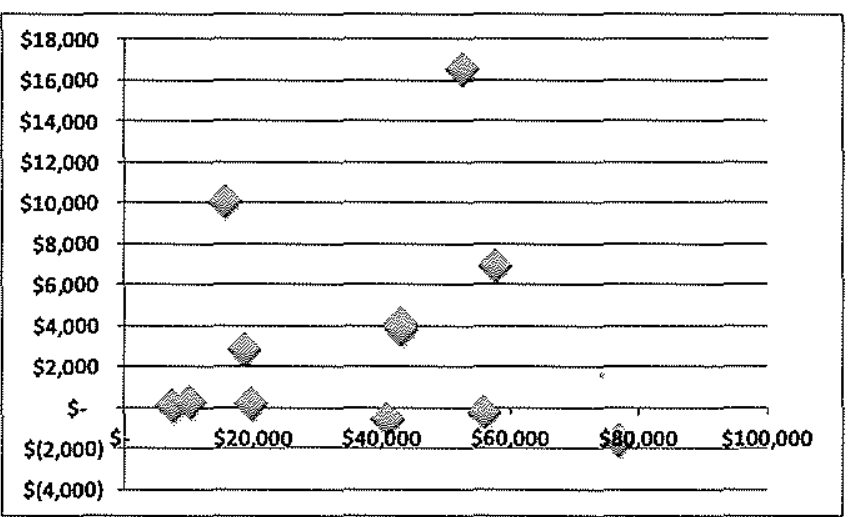

Figure 12. Total Dollars of Systems Engineering Cost Increase Against Total Dollars of Reserves 
Immediately, we encountered a problem with our hypothesis: within our data set, only one mission had total cost increase within the value of the reserves reported at PDR. All twelve other missions spent as much or more than their reserves over the PDR estimate. There did not appear to be evidence that mission cost increases are throttled as reserves run out. However, since the relationship between spacecraft and payload cost increases and reserve levels were also strong, we tested the hypothesis on these two categories in isolation as well. Results were surprising.

We generated a graph showing spacecraft cost increases as a percentage of PDR reserves in order from smallest percentage increase $(-11 \%)$ to largest percentage increase (229\%) (Figure 13). The graph shows that spacecraft costs were increasing at an increasing rate, exactly the opposite of our hypothesis. However, upon closer examination of the graph, we discovered two groups of cost increases which were behaving differently.

1. For missions whose spacecraft increases were less than or equal to reserves (eight total missions), cost increases were behaving as we expected. That is, they were increasing at a decreasing rate. On Figure 13, note that as spacecraft costs as a percentage of total reserves approach 1 , the bars level off, showing that spending for these missions was slowing down.

2. For missions whose spacecraft cost increases exceeded reserves (five total missions), the spending appears to increase at an increasing rate. Figure 13 shows that the data is increasing faster and faster for all the bars over $100 \%$.

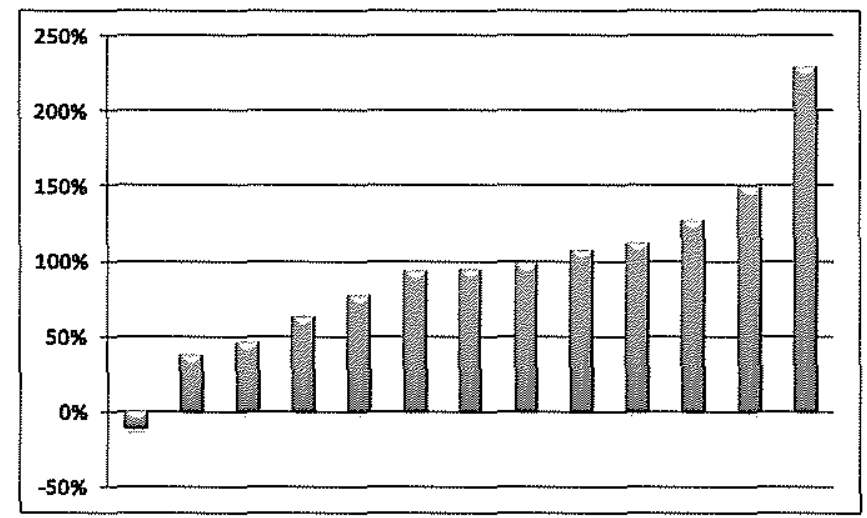

Figure 13. Spacecraft Cost Increase as a Percentage of PDR Reserves, in order from Smallest Percentage Increase to Largest Percentage Increase

To illustrate this, we separated the data into two groupings, shown in Figure 14 and Figure 15.

We tested for a similar pattern with payload cost increases and were unable to establish a similar relationship conclusively. It is interesting to note, however, that payload cost increases also seem to show two separate groupings, those that experience cost increases less than total reserves, and those that experience cost increases greater than total reserves (Figure 16).

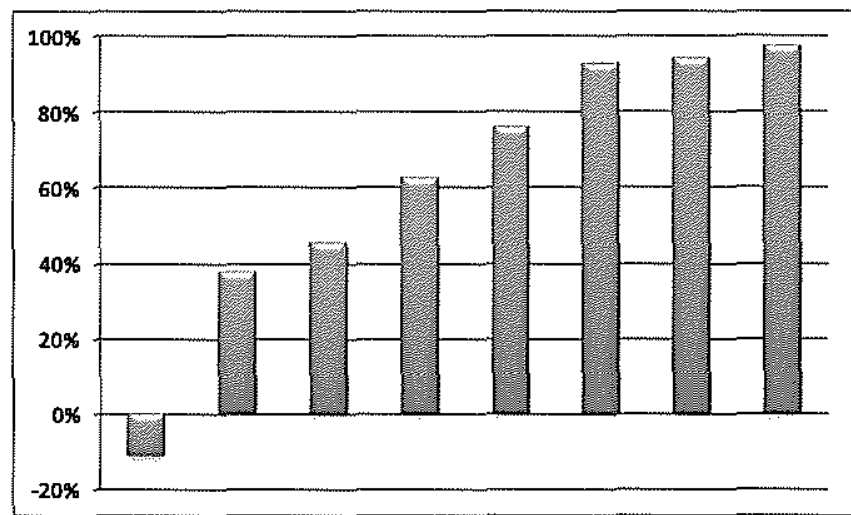

Figure 14. Spacecraft Cost Increase as a Percentage PDR Reserves for all Missions Where Spacecraft Spending Increased Less Than Total Reserve Dollars

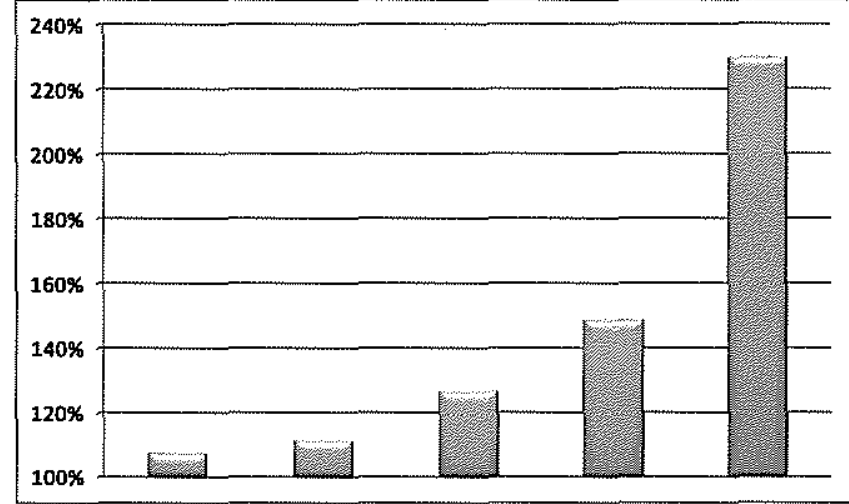

Figure 15. Spacecraft Cost Increase as a Percentage of PDR Reserves for all Missions Where Spacecraft Spending Increased More Than Total Reserve Dollars

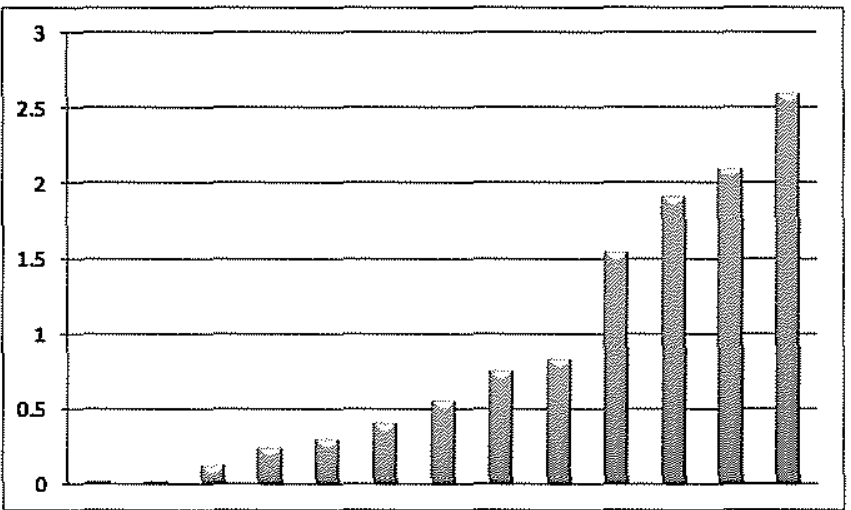

Figure 16. Payload Cost Increase as a Percentage of PDR Reserves, in Order From Smallest Cost Increase to Largest Cost Increase 


\section{Findings, Conclusions AND RECOMMENDATIONS}

Although it was impossible to tell from our limited data set whether the existence of mission cost reserves increases the likelihood of mission cost increases, it was clear from this quantitative research that the amount of mission cost reserves correlates strongly with the amount of mission cost increases. This relationship was also strong between dollars of mission reserves and dollars of spacecraft and payload cost increase.

Furthermore, although the third part of our hypothesis, that mission cost increases will slow as reserve dollars are successively spent (Equation 3), was disproved by our data set, it appears that a qualified version of this part of our hypothesis may yet be valid. For missions where spacecraft cost overruns remain within the amount of mission reserve dollars, overspending does, in fact, appear to be throttled as the spacecraft cost increases approach the total of reserves. However, for missions where spacecraft cost increases exceed total dollars of reserves, spending actually increases at an increasing rate, once reserve dollars are used up.

If we confine our analysis to spacecraft cost increases, the question remains whether the relationship between overspending and reserves is causal. In order to test this, we will need to examine the application of reserve dollars across NASA institutions and investigate the chronology of reserve draw-down. To support the conclusion that the availability of reserves actually causes mission cost increases, we must explore whether "extra" dollars are used to solve project problems as they emerge, to mitigate mission risks, to correct for poorly estimated mission costs at each milestone, or to "gold-plate" a project as it nears its completion.

Although this level of analysis is beyond the scope of this paper (or, more pertinently, it is beyond the scope of our available data set), we will embark on this study in the coming year. Some of the evidence given in this paper already points to the conclusion that the amount of cost reserves does, indeed, bear a causal relationship with the amount of cost overruns, particularly for spacecraft and payload. Assuming this is the case, how can we change reserves policies and practices to reduce the likelihood of generating incentives to overspend?

One solution may be to alter reserve policies so that reserves are calculated as a function of overall mission risk, rather than setting reserves at a fixed percentage of total costs, independent of the specific characteristics that cause a mission to be more or less likely to use reserve dollars. Since reserves are intended to mitigate cost risk, they must be understood in terms of probability and variability. It is reasonable to calculate them as a whole, in terms of some measure of variability of the distribution of summed WBS costs, rather than to calculate them individually by WBS, thus earmarking each dollar of reserves. In light of the expectation that WBS elements within a total mission cost are often correlated with each other, it is important to note that the sum of the most likely outcomes (modes) of the individual cost distributions associated with each WBS element does not equal the mode of the distribution of summed costs (Book, 1999) (Equation 4).

$$
\sum_{i=1}^{n} f_{\text {mode }}\left(W B S_{i}\right) \neq f_{\text {mode }}\left(\sum_{i=1}^{n} W B S_{i}\right)
$$

\section{Equation 4}

What is commonly referred to as the "most likely" outcome of the distribution of mission costs is often significantly lower than the true mode when correlation is taken into account.

Assuming the goal is to achieve $75 \%$ percent confidence that sufficient funds will be available to complete a mission, it makes sense to calculate reserves as the difference between the $75^{\text {th }}$ percentile of the cumulative distribution function of total mission costs and the most likely outcome (mode) of total mission costs (Equation 5).

$$
\begin{aligned}
\text { Reserves } \equiv & F_{0.75}(\text { TotalMissionCosts }) \\
& -f_{\text {mode }}(\text { TotalMissionCosts })
\end{aligned}
$$

\section{Equation 5}

This allows cost estimators to calculate mission costs as the outcome of the cost distribution that has the highest probability of occurring, and to quantify cost risk as the dollar amount required to achieve an adequate level of confidence in the mission's viability.

This approach is not unheard of. Statistical cost estimating techniques for space missions rely on extremely sparse data sets with broad definitions of similar mission characteristics. For example, in order to achieve a reasonably broad sample for this paper, we defined our data set as NASA missions launched since 2004 with at least two CADRe data entries. For this paper, as well as for any analysis used to estimate expected mission costs, the cost distributions are therefore often heavily right-skewed with very fat tails. With this type of data, measures of expected value are often estimated from the median or the mode of the distribution, rather than from its mean (Fleming, 2007). Adding an uncertainty component equal to the distance from the measure of central tendency to a particular percentile of the cumulative distribution function is a commonly used method of mitigating risk in insurance pricing (Miscellaneous insurance rate-setting paper I'll dig up and cite).

We have shown that reserve spending is currently (at least in part) a function of reserves. We have also provided strong evidence that reserve spending can actually be caused by the presence of reserves. We have offered a possible solution in the form of redefining reserves as a function of cost risk. That is, a project should directly assess Technology Readiness Level (TRL), complexity of missions, process 
maturity levels, and institutional competencies. But, how does this align incentives so that organizations are less likely to use reserves? By institutionalizing this approach, we could examine if reserves are less likely to be spent in this project formulation. Limited data availability inhibits the complete study of whether knowledge of reserve levels can cause a different spending behavior. But, if a project (and stakeholders) were to estimate reserve necessity based purely on the critical elements listed above, projects could then generate a meaningful reserve assessment.

\section{REFERENCES}

Akerlov, George. "The Market for Lemons: Quality Uncertainty and the Market Mechanism." Quarterly Journal of Economics Volume ?(1970): p.?.

Author. "Title." p. ?, ?, Volume ?, Issue ?, Year?. Miscellaneous Rate Setting Paper from CAS Exam 5 , presumably in my big pile of old actuarial exam stuff; I'll find it.

Bergemann, Dirk and Ulrich Hege. "Venture Capital Financing, Moral Hazard, and Learning." Journal of Banking and Finance Volume 22 (1998): Issues 6-8.

Book, Stephen A. "Why Correlation Matters in Cost Estimating." $32^{\text {nd }}$ Annual DOD Cost Estimating Symposium (1999).

Emmons, Deborah L., Robert E. Bitten, and Claude W. Fraener. "Using Historical NASA Cost and Schedule Growth to Set Future Program and Project Reserve Guidelines." 2010 IEEE Aerospace Applications Conference Proceedings (2010): p.?.

Fleming, Kirk G. "Yep, We're Skewed." Variance Volume ? (Year?): p.?.

Mirrlees, ?. "The Theory of Moral Hazard and Unobservable Behavior." Review of Economic Studies Volume 66 Issue 1 (1999): pp. 3-21.

Office of the Chief Engineer. NPR 7120-81 NASA Space Flight Program and Project Management Requirements NASA, (2009): p. ?.

Prescott, E.C. "Pareto optima and competitive equilibria with adverse selection and moral hazard." Econometric Volume 52 No. 1 (1984): p.?

Shinn, Stephen A., et al. "Update: Growth of Robotic Space Missions System Engineering and Program Management Costs." 2010 IEEE Aerospace Applications Conference Proceedings (2010): p. ?,
Spear, S.E. "On Repeated Moral Hazard with Discounting." Review of Economic Studies Volume 54 No. 4 (1987): p.?

\section{ACKNOWLEDGeMENTS}

$? ?$ 